\title{
Paediatric cardiac computed tomography: a review of imaging techniques and radiation dose consideration
}

\author{
Carolyn Young • Andrew M. Taylor • \\ Catherine M. Owens
}

Received: 26 November 2010 /Revised: 6 December 2010 /Accepted: 8 December 2010 /Published online: 28 December 2010

(C) European Society of Radiology 2010

\begin{abstract}
The significant challenges involved in imaging the heart in small children $(<15 \mathrm{~kg})$ have been addressed by, and partially resolved with improvement in temporal and spatial resolution secondary to the advent of new multidetector CT technology. This has enabled both retrospective and prospective ECG-gated imaging in children even at high heart rates (over $100 \mathrm{bpm}$ ) without the need for beta blockers. Recent studies have highlighted that the radiation burden associated with cardiac CT can be reduced using prospective ECG-gating. Our experience shows that the resultant dose reduction can be optimised to a level equivalent to that of a non-gated study. This article reviews the different aspects of ECG-gating and the preferred technique for cardiac imaging in the young child $(<15 \mathrm{~kg})$. We summarize our evidenced based recommendations for readers, referencing recent articles and using our in house data, protocols and dose measurements discussing the various methods available for dose calculations and their inherent bias.
\end{abstract}

Keywords Paediatric - Cardiac CT · Retrospective ECGgating $\cdot$ Prospective ECG-gating $\cdot$ Radiation dose

C. Young $\cdot$ A. M. Taylor $\cdot$ C. M. Owens

Cardiorespiratory Unit, UCL Institute of Child Health,

London, UK

C. Young $(\bowtie) \cdot$ A. M. Taylor

Cardiorespiratory Unit, Great Ormond Street

Hospital for Children,

Great Ormond Street,

London WC1N 3JH, UK

e-mail: YoungC@gosh.nhs.uk

\section{Introduction}

Innovations in computed tomography (CT) technology with improved spatial and temporal resolution have extended the application of cardiovascular imaging to encompass paediatric patients with complex congenital heart disease (CHD). Multi-detector CT (MDCT) led the way enabling imaging of cardiovascular structures and coronary artery anomalies in children without the use of ECGsynchronisation [1-4]. However, greater accuracy in coronary artery anatomical delineation was seen using retrospective ECG-gating, and was subsequently applied to CT imaging of neonates with CHD [5, 6]. However the increase in radiation burden associated with retrospective ECG-gating has been a limiting factor that has reduced uptake in paediatrics. Conversely the use of prospective ECG-gating with its much reduced radiation dose, has always been confined to adult patients with low heart rates [7], with studies demonstrating no significant difference in image quality or coronary artery detection rate between prospective and retrospective ECG-gated imaging [8-12].

With the advent of dual-source (DSCT) technology (Siemens Definition, Forchheim, Germany) it is now feasible to extend ECG-synchronised cardiac imaging (utilising both retrospective and prospective gating) to the paediatric population even for non-coronary applications [13-16], (Fig. 1). Faster volume coverage together with higher temporal resolution (75-83 ms), mean that high heart rate are no longer a limiting factor for $\mathrm{CT}$ in children and adults $[5,17]$ using prospective gating - much reduced radiation dose with image quality maintained [18-20] (Fig. 2). 
Fig. 1 Retrospective

ECG-triggered study on a 2-month-old child with a heart rate of $130 \mathrm{bpm}$ investigated for incomplete closure of VSD and duplication of the aortic arch, shown on coronal MIP (image a) and on the VRT images (b \& c). The coronary arteries are clearly seen in the reformatted images $\mathbf{d} \& \mathbf{e}$. CTDIvol $=2.65 \mathrm{mGy}$, effective dose $=2.7 \mathrm{mSv}$
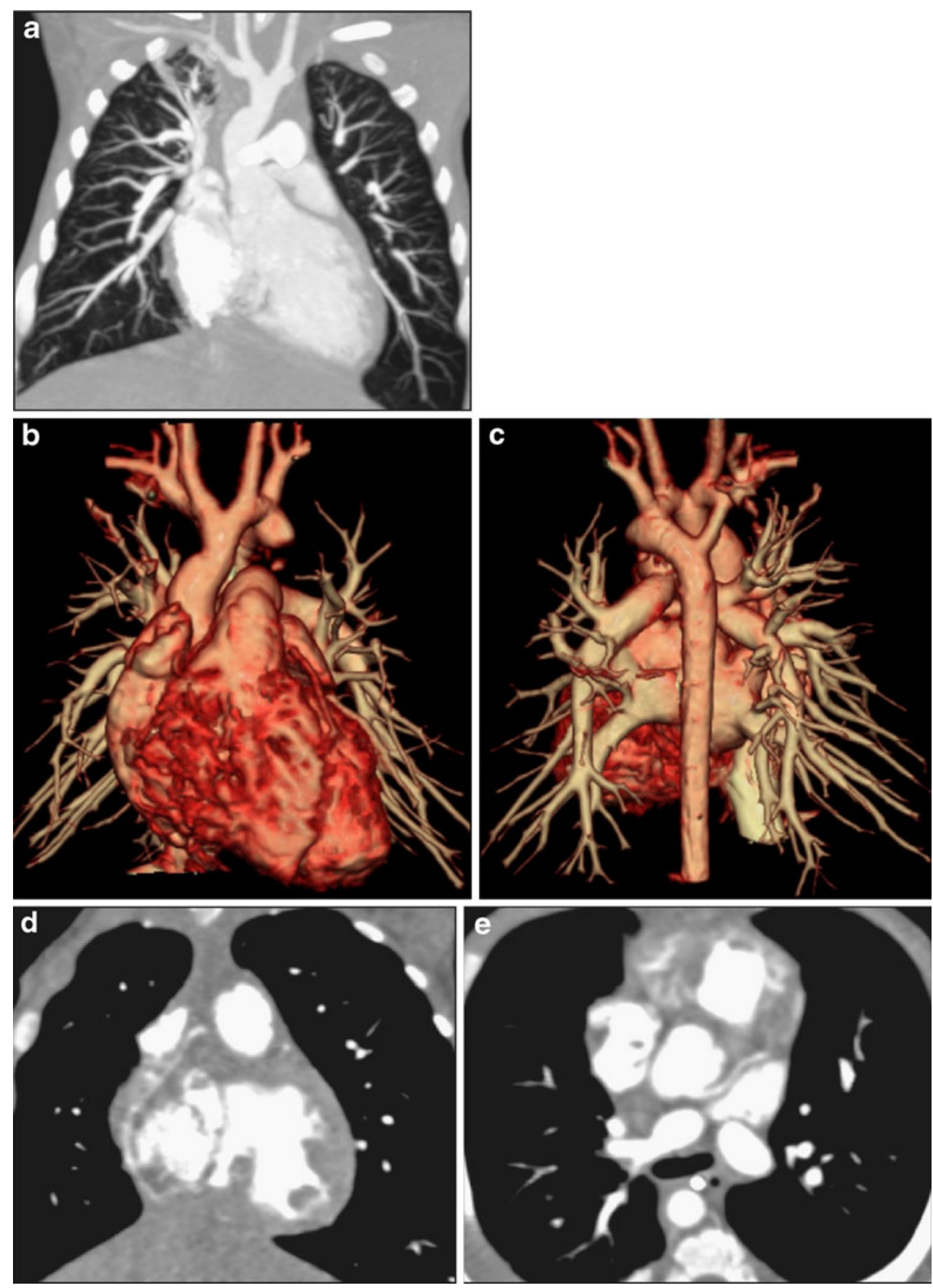

\section{Non-ECG-synchronised CT angiography}

Spiral non-gated MDCT is a widely used imaging technique in the evaluation of thoracic and cardiovascular malformations in young children $[4,5,19]$. Its capability to acquire sub-millimetre isotropic data has minimised respiratory and motion artefacts with improved image resolution. The ability to view and manipulate reconstructed image data in different orthogonal planes has enhanced diagnostic accuracy $[4,21,22]$.
Even without ECG-gating, good visualisation of the proximal coronary artery has been reported in a study utilising 16-slice MDCT [3]; however clarity of the coronary arteries was found to be age and heart rate dependent, Fig. 3. Where greater definition is required for assessing coronary arteries in patients with complex CHD, Ben Saad et al. [5] and Goo [19] have devised a 'Combo' $\mathrm{CT}$ protocol. This includes a non-gated spiral examination of the entire thorax followed by a limited ECG-triggered DSCT focusing on the conotruncal region. For this second 
Fig. 2 Prospective

ECG-triggered study on a 1 week old $(1.8 \mathrm{~kg})$ neonate with pulmonary atresia (images a, b). Image quality was not affected by a high heart rate of $123 \mathrm{bpm}$, with good visualisation of the coronary arteries (images $\mathbf{d}, \mathbf{e}$ ).

CTDIvol $=1.18 \mathrm{mGy}$, effective dose $=0.83 \mathrm{mSv}$
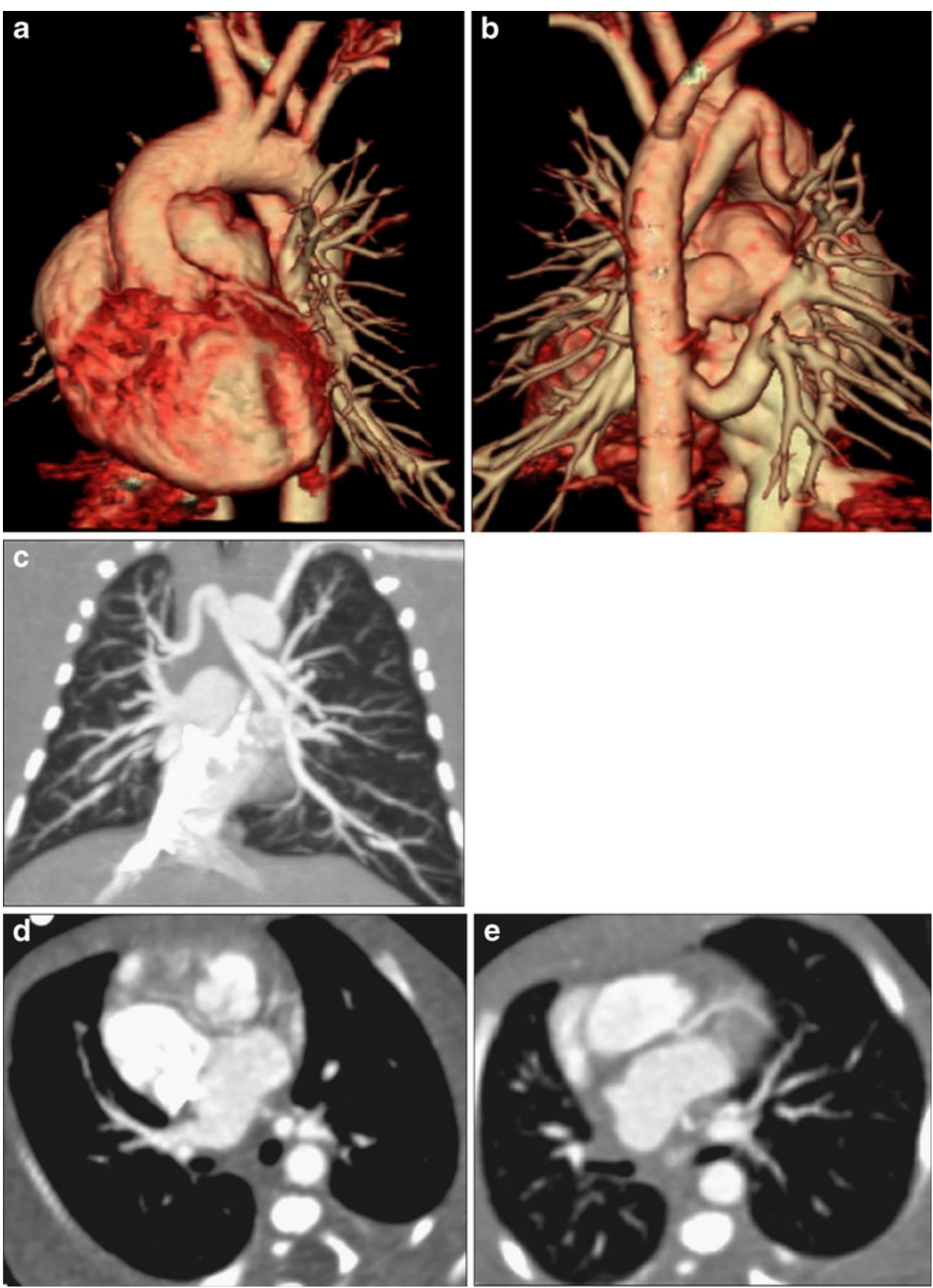

phase, Saad has adopted the retrospective gating methodology with Goo opting for prospective gating. The reported combined doses are 1.8 and $1.4 \mathrm{mSv}$ respectively in children under 1 year of age.

As with all spiral acquisitions in $\mathrm{CT}$, there is an inevitable increase in radiation dose due to the longitudinal over-ranging at both ends of coverage [14]. This problem has been addressed by manufacturer with incorporation of adaptive dose shields to block radiation during the 'run-up' and 'run-down' time necessary for spiral data acquisition (Siemens Healthcare, Forchheim, Germany).
Imaging technique

Unlike adult cardiac imaging, CT angiography coverage in paediatric CHD patients extends from the chest apices to the dome of the diaphragm dependent on clinical indications [19], and often with extended infra-cardiac coverage in the assessment of anomalous pulmonary venous return or aorto-pulmonary collateral vessels (Fig. 4). Adequate visualisation of the proximal coronary artery origin is also possible [3, 19], with the left coronary artery better defined than the right coronary artery (Fig. 5). 
Fig. 3 3.1 Image sharpness is degraded due to motion artefact in this non-gated study on an 8-month-old (2.2 kg.) ex premature baby with chronic lung disease. CTDIvol $=6.2 \mathrm{mGy}$, effective dose $=0.54 \mathrm{mSv}$. 3.2 The LCA is clearly demonstrated in image a in this non-gated study on a 5-year-old child with left upper lobe bronchial narrowing and emphysema. The origin of the RCA is clearly seen on image b. CTDIvol=1.53 mGy, effective dose $=1.4 \mathrm{mSv} .3 .3$ Chest study on a 12 -year-old patient with interstitial lung disease. There is good opacification of the LCA seen in the processed MIP image a. However the RCA is not clearly defined on image $\mathbf{b}$. CTDIvol $=4.31$, effective dose $=3.5 \mathrm{mSv}$
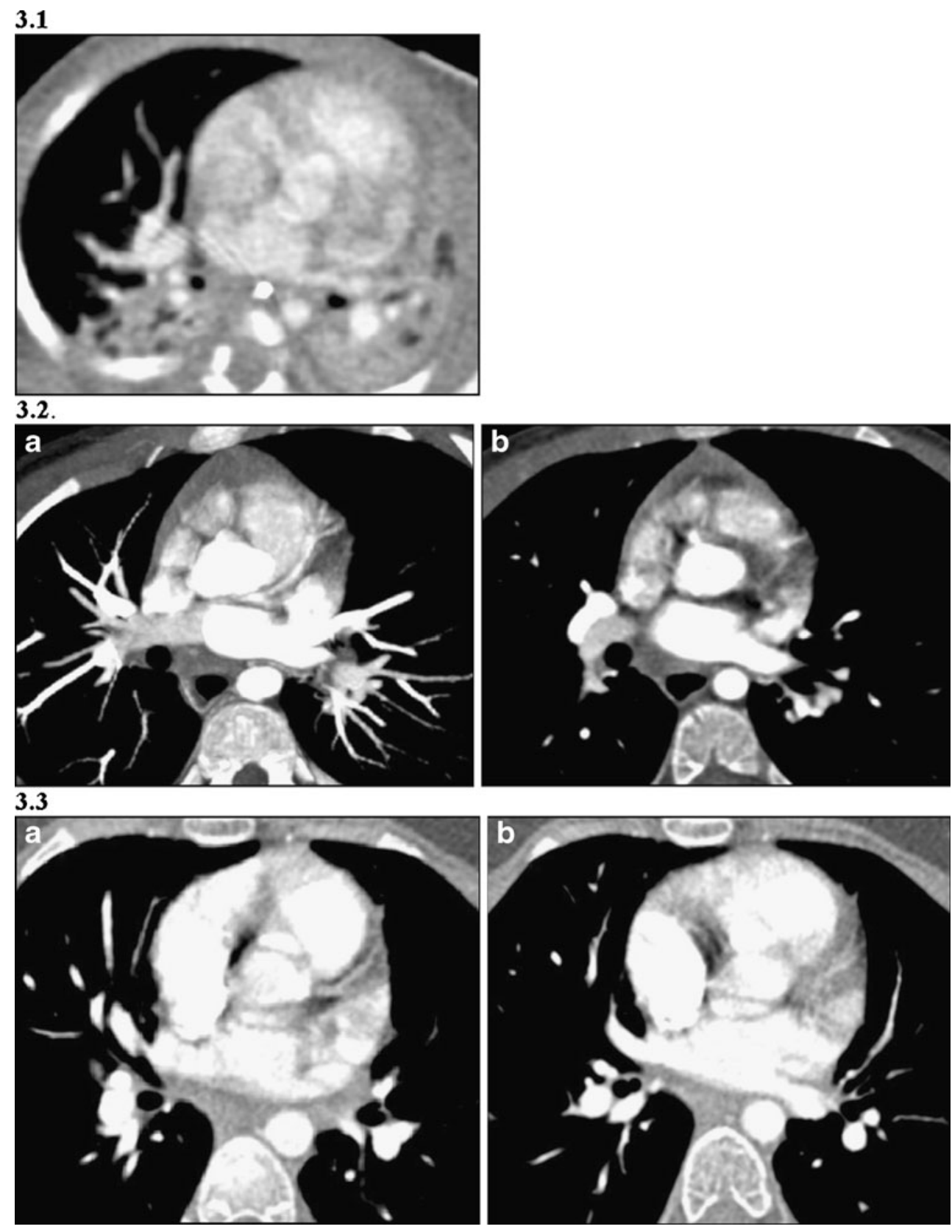

When imaging younger children, a thin slice collimation should be used although this does increase image noise. This can be overcome by increasing the reconstructed slice thickness (but results in spatial resolution reduction) by using multi-planar reformats.

A pitch of 1 maintains the balance between good image quality and a reasonable dose level. Although a pitch greater than 1 reduces CT data acquisition time, it is not advocated due to degradation of longitudinal spatial resolution whilst increasing radiation dose for those systems that utilise effective mAs. However this method is adequate for gross pathology assessment in noncompliant patients [23], (Fig. 6).

\section{Retrospective ECG-gated spiral CT}

Cardiac imaging with retrospective ECG-gating is widely used in adults, utilising both single source MDCT (SSCT) and more recently dual-source technology. To ensure good temporal resolution in SSCT, a low pitch (0.2) with multisegment reconstruction is required. The use of a low pitch slows the table advancement to less than the detector's width per tube rotation (overlapping slices), resulting in the same chest region being exposed multiple times, increasing radiation burden and prolonging total acquisition time. Together with the inability to apply ECG-gating at heart rates above $80 \mathrm{bpm}$, this limits this application of retrospective 

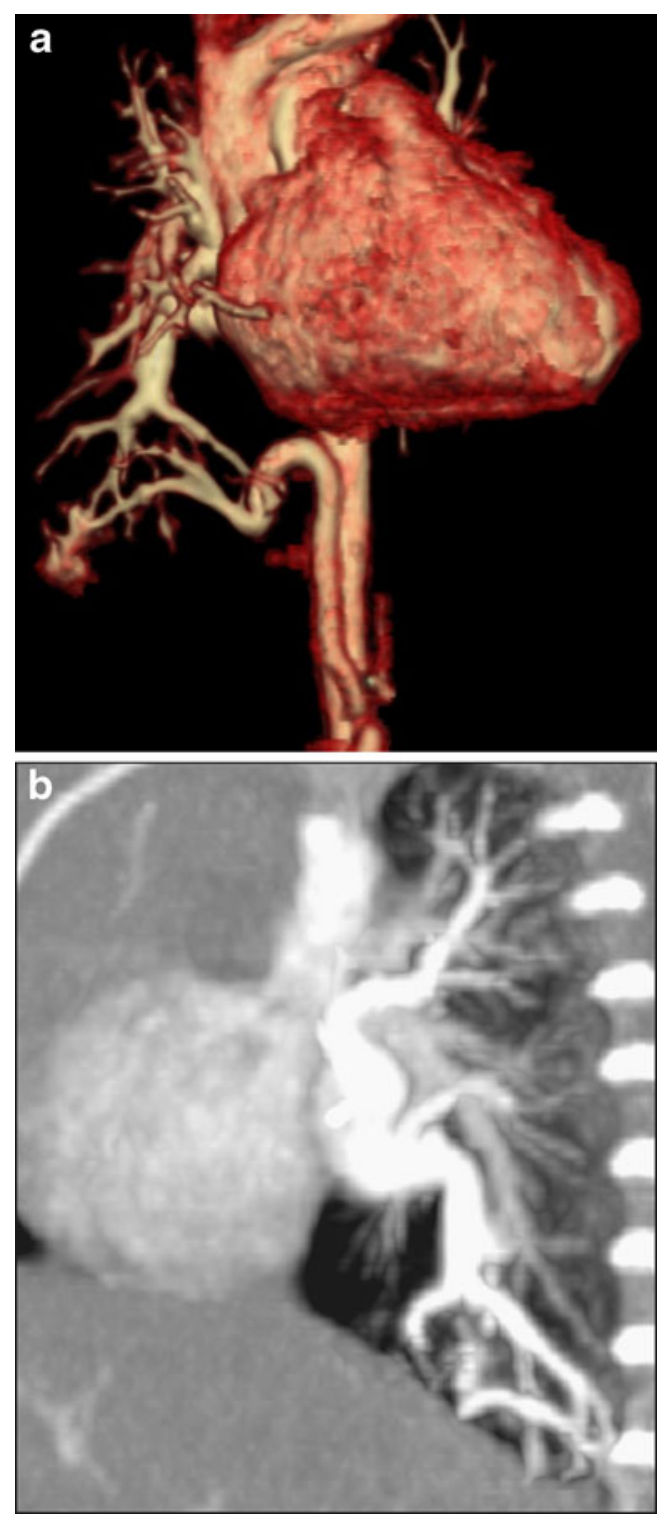

Fig. 4 Volume rendered images of a CTA study on a non-sedated 1-month-old child, shows the importance of extended coverage required in suspected cases of congenital pulmonary airway malformation (CPAM)/Sequestration. The images demonstrate a systemic arterial supply (a) originating from the anterior aorta (located between the coeliac axis and SMA) with a large draining vein (b) connecting to the right inferior pulmonary vein. CTDIvol $=0.61 \mathrm{mGy}$, effective dose $=0.9 \mathrm{mSv}$

gating in children. In DSCT however, with single segment reconstruction and improved $83 \mathrm{~ms}$ temporal resolution (irrespective of heart rate), evaluation of rapidly moving cardiac structures without the need for beta-blockers is possible. Its usage is also contra-indicated in children as it may be insufficiently effective or even hazardous.

Radiation dose saving features of retrospective DSCT includes an adaptive pitch that increases with increasing heart rate, together with ECG controlled tube-current modulation. This has the potential for lower radiation dose with image quality maintained even at high heart rate [5,
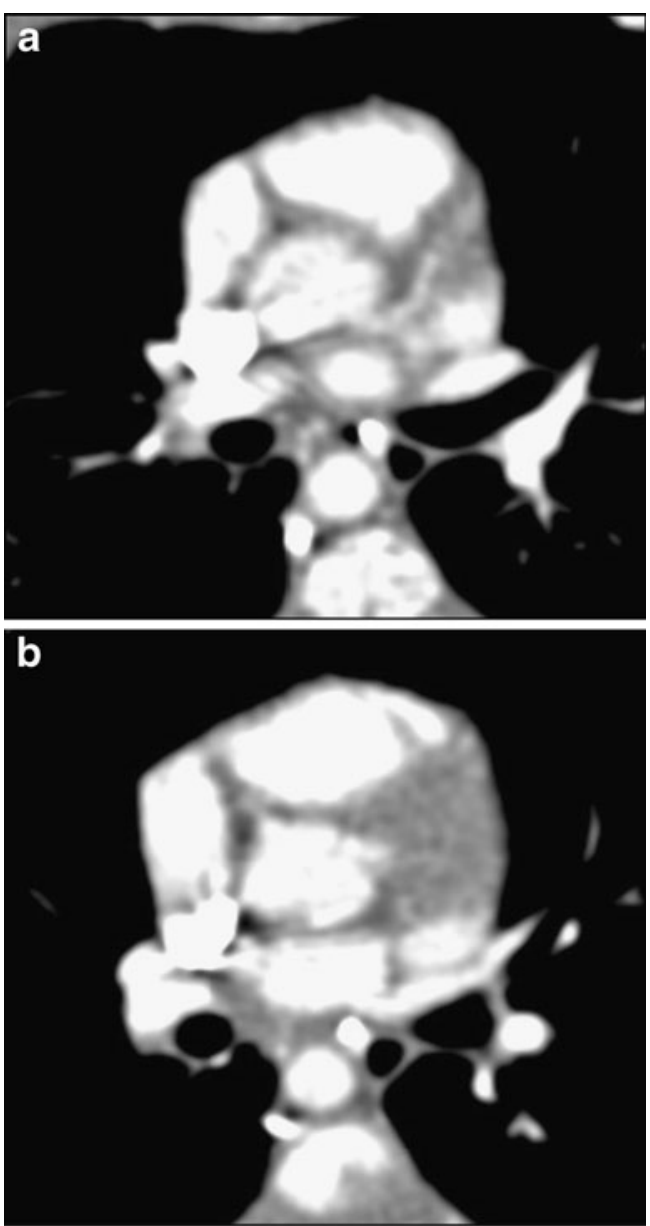

Fig. 5 A non-gated CTA study in a 3-month-old child that shows good opacification of the proximal LCA (image a), but RCA not clearly defined on image b. CTDIvol $=0.68 \mathrm{mGy}$, effective dose $=0.76 \mathrm{mSv}$

23], making this a reliable imaging modality for the evaluation of complex congenital abnormalities in children.

Imaging technique

In retrospective ECG-gated CT, data acquisition continues throughout the cardiac cycle with maximum tube current applied across the diastolic phase and a modulated tube current during the rest of the cardiac cycle. In children, the end systolic phase (where maximum tube current is applied) is preferred with HR above $80 \mathrm{bpm},[7,14,19,20]$ this being less susceptible to motion artefacts, (Fig. 7). Determining an optimal temporal window width where maximum tube current is applied is dependent on diagnostic information required and varies with different heart-rate - a narrow window for low heart rates and wider at high heart rates.

There are two levels of ECG-controlled tube current modulation available, whereby the tube output can be reduced to $25 \%$ or $4 \%$ (min-dose, Siemens Healthcare, Forchheim, Germany) outside the predefined temporal window [7, 8, 20]. The former (i.e. $25 \%$ ) with partial loss of image quality is 

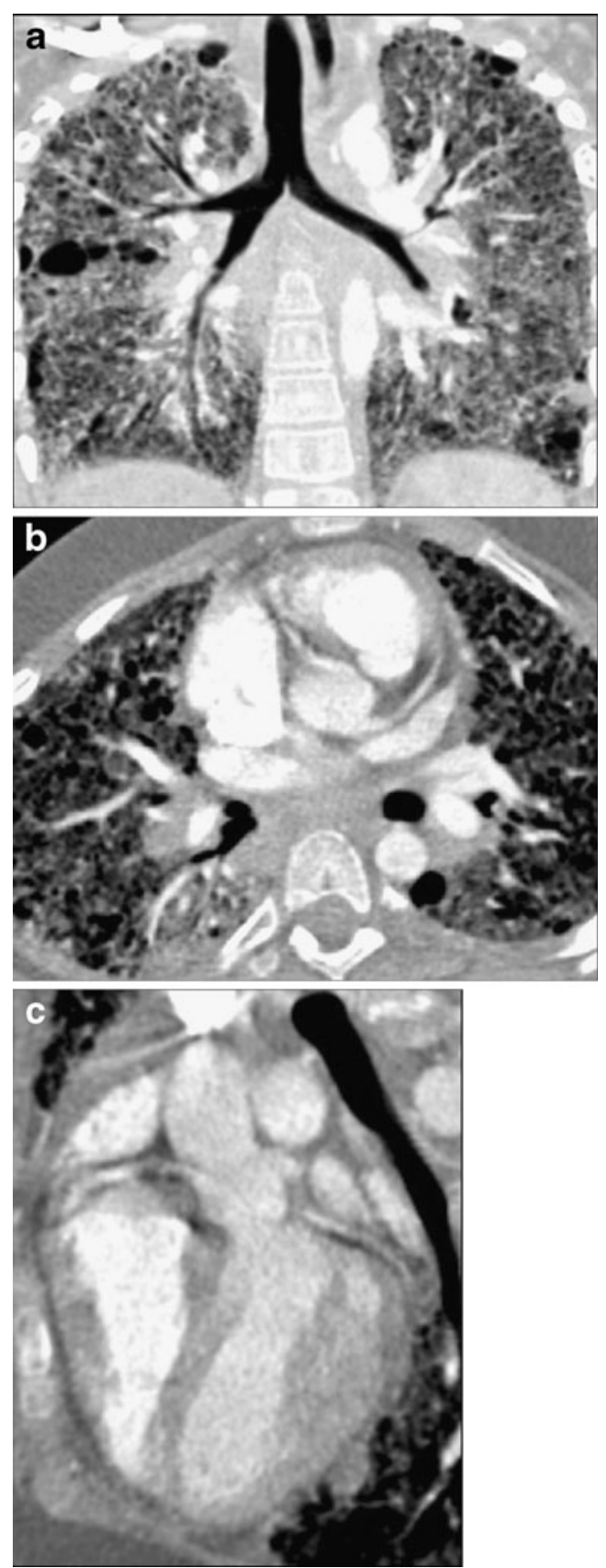

Fig. 6 6-year-old child underwent a non-gated study for the assessment of known chronic lung disease and pulmonary arterial hypertension. Due to extreme shortness of breath, a pitch of 1.5 was used to shorten the acquisition time. Image quality is adequate for gross pathology as demonstrated in image a, with good visualisation of the coronary arteries, images b \& c. CTDIvol $=2.54 \mathrm{mGy}$, effective dose $=2 \mathrm{mSv}$

still sufficient for functional analysis but a reduction to $4 \%$ may only be limited to imaging where gross overall anatomical evaluation is necessary [14]. Our experience is that the degree of dose reduction is heavily dependent on the applied CT parameters. Reduction to $4 \%$ cannot be achieved if exposure factors used are already at the lower limitations of the x-ray generator, so this will prevent further reduction in output.
This is confirmed by phantom studies and acknowledged by the relevant manufacturer.

DSCT allows adaptation of table feed to heart rate, i.e. pitch increases with increasing heart rate from 0.2 to 0.5 at $100 \mathrm{bpm}$. At a higher pitch, CT data acquisition time is reduced with fewer requirements for overlapping slices. This results in a dramatic dose reduction [23].

\section{Prospective ECG-gated sequential CT}

Prospectively ECG-triggered sequential MDCT is a well recognised diagnostic tool for the evaluation of coronary artery disease in adults at low and stable heart-rates. It is also an efficient technique for dose reduction characterised by applying maximum tube current at a pre-defined phase in the R-R cycle, with complete cessation of radiation output during the remainder of the cardiac cycle as the imaging couch moves to the next position in the $\mathrm{z}$ direction.

DSCT enables the use of prospective ECG-gated sequential $\mathrm{CT}$ in children, even at high heart rates over $100 \mathrm{bpm}$, utilising the on-line ECG to trigger data acquisition at every 3rd heart beat, (Fig. 8). The high temporal resolution has the ability to 'freeze' cardiac motion, whilst spatial resolution is maintained, (Fig. 9).

Compared with retrospective gated imaging there are limitations associated with this adaptive sequential CT technique that include: i) Ventricular functional analysis cannot be obtained; ii) Possible difference in contrast enhancement between adjacent slice slabs, (Fig. 10); iii) Increased step artefacts when compared to retrospective gating due to movement in non-compliant patients, (Fig. 11); iv) Prolonged data acquisition time that may cause respiratory artefact if breath-hold cannot be maintained for that length of time [20], (Fig. 12).

Imaging technique

It is advocated when performing cardiovascular CT on children with heart rate above $80 \mathrm{bpm}$, that data is acquired at end-systole using an absolute (ms) trigger delay as opposed to a relative (percentage) trigger delay [8, 18-20] to ensure maximum image quality. This will also help minimise stair-step artefact. This step artefact is not problematic when interpreting axial slices, only being more pronounced when displayed in the different orthogonal planes, as shown in Figs. 10 and 11.

User-defined acquisition window on the DSCT enables either a $330 \mathrm{~ms}$ acquisition with extended imaging angle from $260^{\circ}$ to $460^{\circ}$, which results in a $\pm 8 \%$ phase shift enabling flexibility in choosing different cardiac phase reconstruction retrospectively [18], i.e. in a neonate with a 
Fig. 7 7.1 Both proximal coronary arteries are clearly seen in the systolic phase of this retrospective ECG-triggered study in a 1-year-old child with a heart rate of $133 \mathrm{bpm}$. CTDIvol= 1.73 , effective dose $=1.6 \mathrm{mSv}$. 7.2 Good opacification of the coronary arteries are seen in the systolic phase (image a) of this retrospective ECG-triggered study on a 2-year-old child with heart rate of $107 \mathrm{bpm}$. On the diastolic phase (image b), the RCA is not seen. CTDIvol= $2.74 \mathrm{mGy}$, effective dose $=$ $2.5 \mathrm{mSv}$
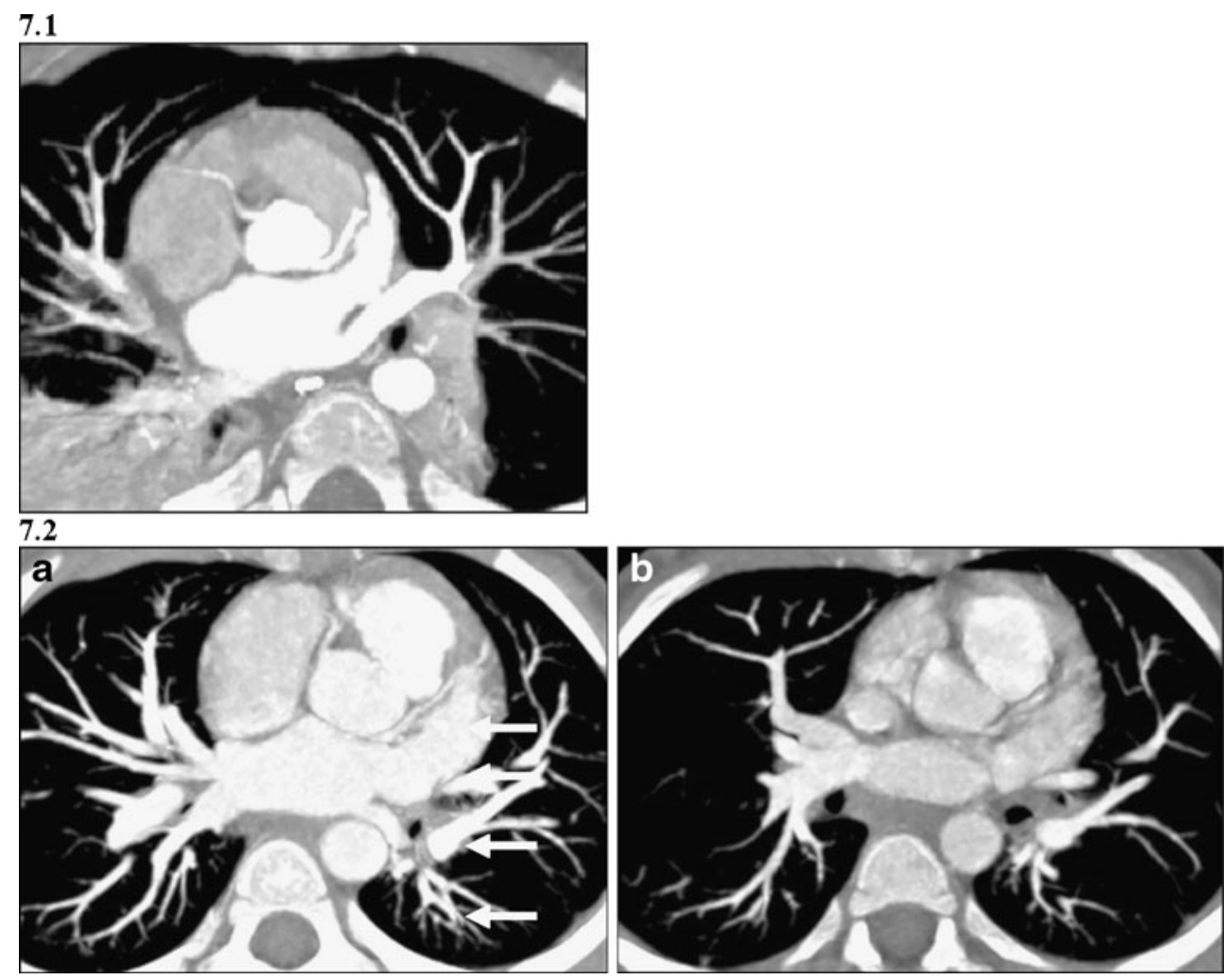

$505 \mathrm{~ms}$ R-R cycle time. This equates to possible reconstruction window between $170 \mathrm{~ms}$ and $350 \mathrm{~ms}$ when the acquisition is set to end-systole. Or choosing the 'quick-step' acquisition window of $220 \mathrm{~ms}$ reduces acquisition time but without the flexibility for different phase reconstruction, thus selection of optimal acquisition phase is crucial.

The contrast layering effect often seen between adjacent acquisition slabs is due to the time delay required for table movement between slices, instead of acquiring data at every heart beat, in children with high heart-rate, data is acquired at every 2 nd or 3 rd heart beat, (Fig. 8). To minimise this effect, the rate of contrast medium delivery should be adjusted to ensure a constant prolonged peak vascular enhancement throughout the CT data acquisition.

\section{Intravenous contrast media}

The concentration of contrast medium used and delivery methods vary between different authors. The amount of contrast medium prescribed was dependent on body weight, varying between 1 and $3 \mathrm{mls}$ per $\mathrm{kg}$ with iodine concentration of between 300 and $400 \mathrm{mg} \mathrm{I} / \mathrm{ml}$ and a flow-rate of between 0.5 and $1.5 \mathrm{ml}$ per second. Intrave-
Fig. 8 An ECG-trace with an acquisition triggered on every third cardiac cycle

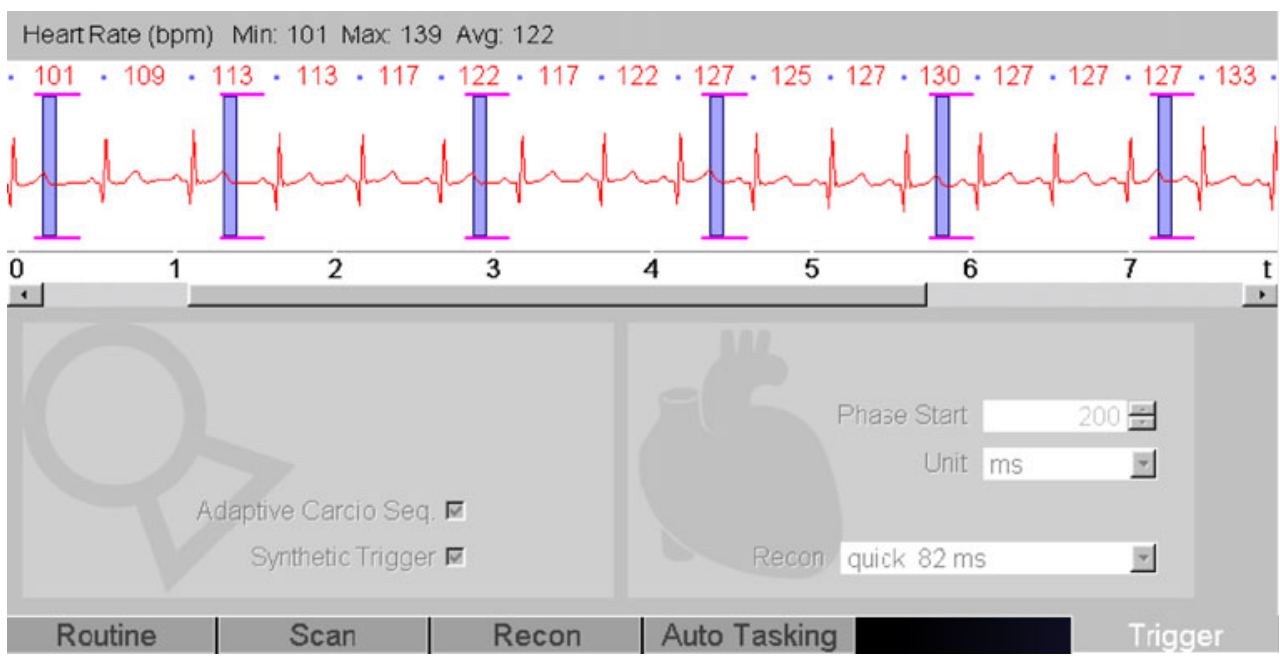



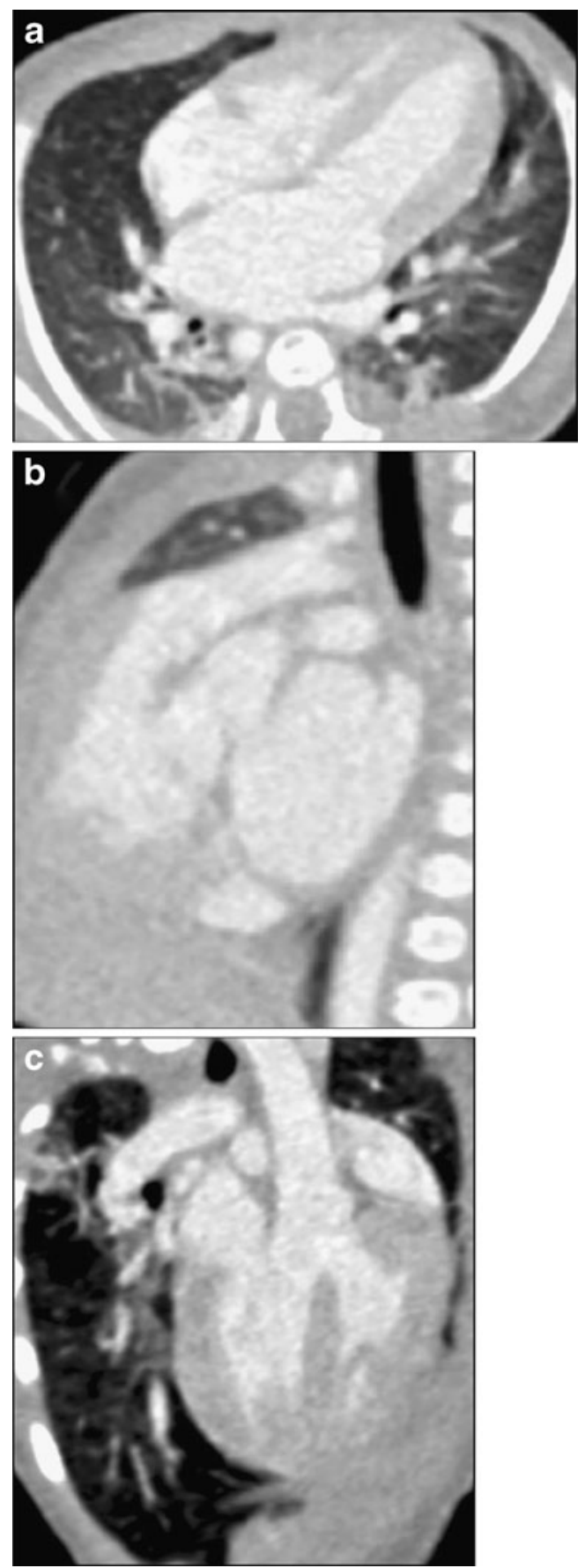

Fig. 9 Prospective ECG-triggered study in a non-sedated, freebreathing 2-month-old child with Tetralogy of Fallot. At a high heart-rate of $137 \mathrm{bpm}$, no degradation in image quality was noted. CTDIvol $=1.22$, effective dose $=0.82 \mathrm{mSv}$

nous delivery of contrast medium using a bolus tracking method was used by Goo \& Yang [19] and Tsai et al. [6], whilst other paediatric centres prescribe a fixed delay before initiating CT data acquisition, to avoid additional radiation from the monitoring imaging. Ben Saad at al. [5] and Paul et al. [24] employed a $15 \mathrm{~s}$ delay in cases where there is peripheral access and $10 \mathrm{~s}$ delay for central venous
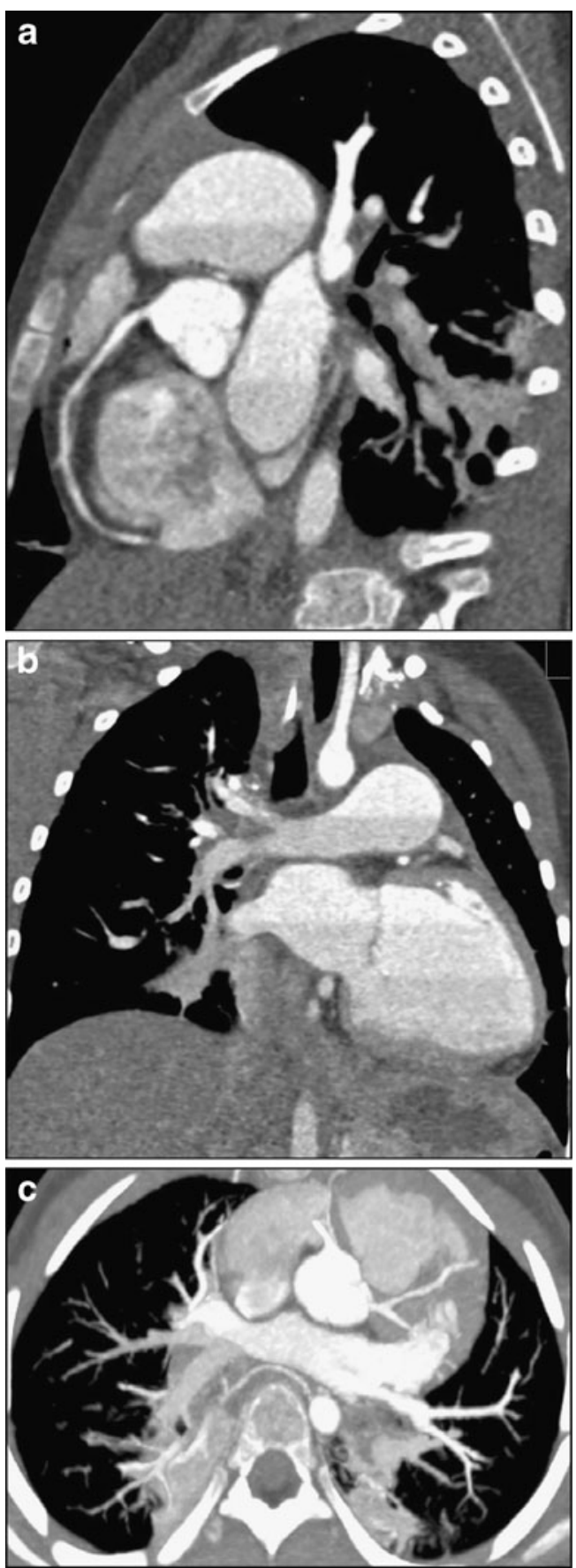

Fig. 10 Sagittal \& coronal images $(\mathbf{a}, \mathbf{b})$ show degradation of image quality due to contrast layering effect in this prospective ECGtriggered study on a 6.5 -year-old child (heart rate $59 \mathrm{bpm}$ ). However, the images are of diagnostic quality with good contrast enhancement seen in the coronary artery (image a) and in the MIP axial image (c). Dilatation of branch pulmonary artery (image b) with collateral vessels (image c) is seen as a consequence of pulmonary arterial hypertension. CTDIvol $=2.81 \mathrm{mGy}$, effective dose $=2.2 \mathrm{mSv}$

access, whilst Cheng et al. [18] applied a fixed $25 \mathrm{~s}$ delay, and Jin et al. [19] used an estimated time of peak enhancement in the targeted vessel minus half the duration of the CT data acquisition. 
Fig. 11 Post-operative prospective ECG-triggered study following BT shunt procedure in a non-sedated 1 month old child, heart rate was $164 \mathrm{bpm}$. Image a shows step artefact distorting the course of the BT shunt due to patient movement. But the study was diagnostic demonstrating good filling of the pulmonary arteries from the BT shunt seen in the coronal images $\mathbf{b} \& \mathbf{c}$ and in the axial MIP image d. CTDIvol $=1.33 \mathrm{mGy}$, effective dose $=0.87 \mathrm{mSv}$
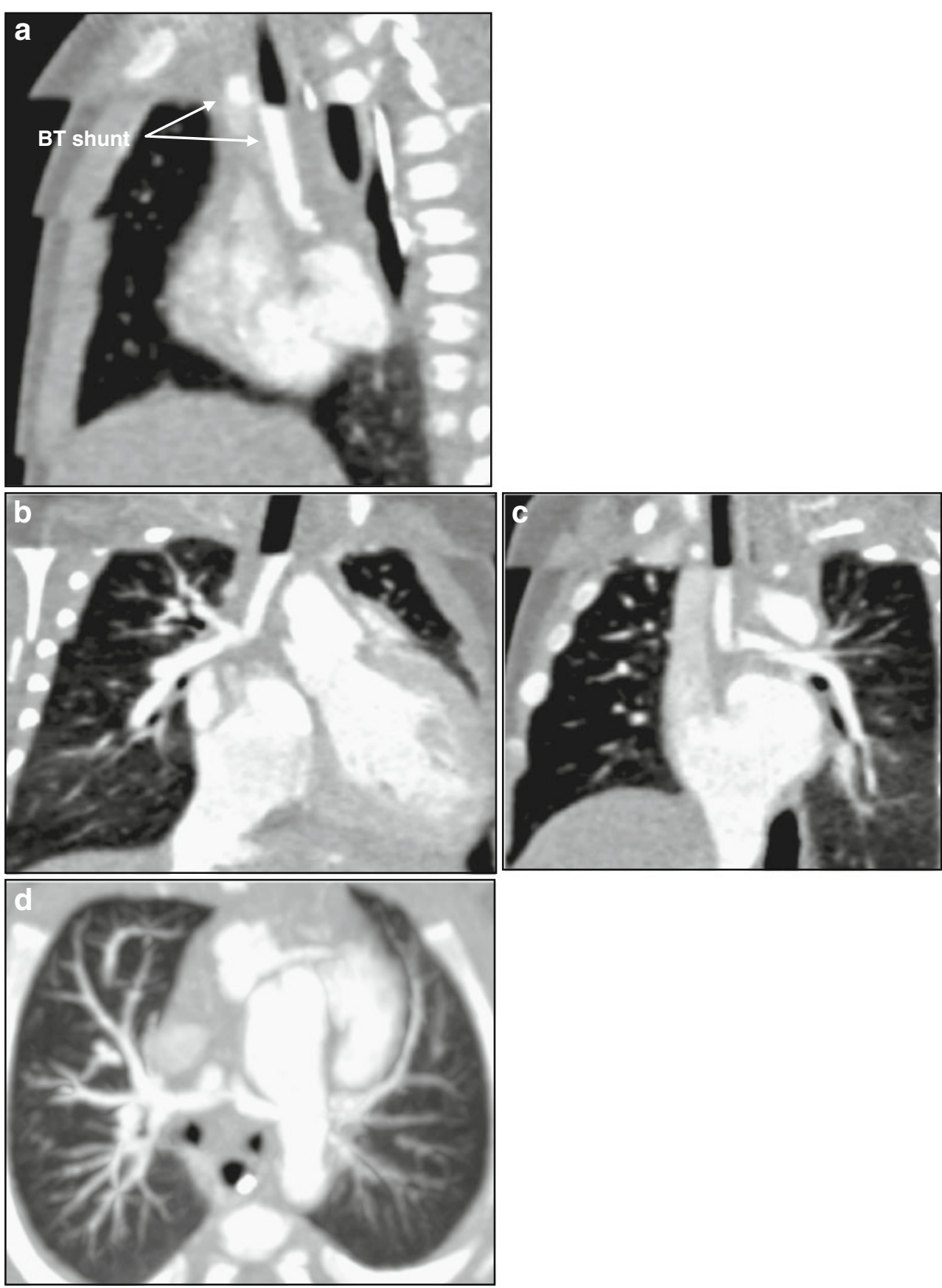

As diagnostic information is not required from the monitoring slices, the mAs can be reduced drastically (to $20 \mathrm{mAs}$ ), and increasing the time delay between each monitoring slice will keep dose to a minimum. Our experience shows the monitoring CT only incur an additional 1DLP (dose length product) to the overall dose.

\section{Radiation dose}

Radiation is a major inherent limitation for ECG-gated cardiac imaging. However, the benefits of this non-invasive diagnostic technique outweigh the potential radiation risks especially when comparing this to a diagnostic cardiac catheter angiography study that carries an additional procedural risk [7].

Dose saving measures are enhanced by employing patient specific weight-based protocols with adaptation of $\mathrm{kVp}$ and mAs: the main determinate of radiation dose received $[1,2,5]$. From the articles reviewed, the standard $\mathrm{CT}$ protocol of $80 \mathrm{kV}$ was used throughout with the mAs adjusted to individual's weight.

Applying tube current modulation reduces radiation dose further by adjustment of the $\mathrm{mA}$ to patient diameter 

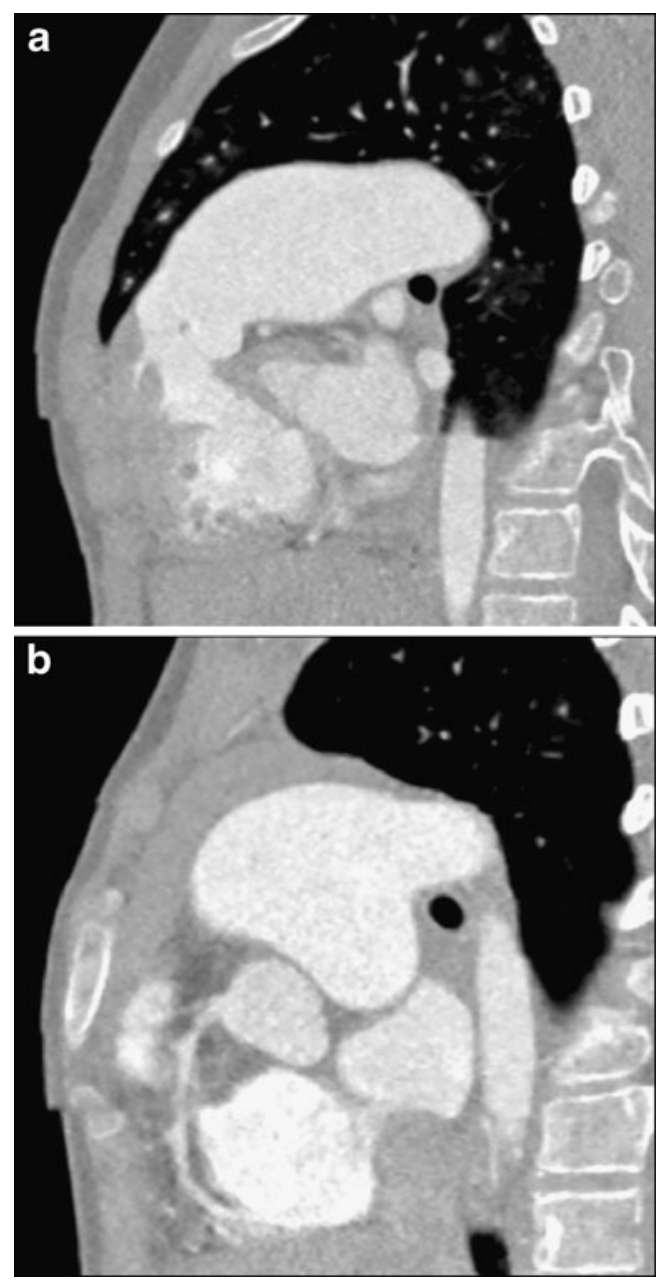

Fig. 12 Respiratory artefacts are present in this prospective ECGgated study in a 13-year-old patient unable to breath-hold due to a prolonged (16 s) CT data acquisition. The artefacts were not so marked as to degrade images that depict the RCA in its entirety (image b). Heart rate was $69 \mathrm{bpm}$. CTDIvol $=2.70 \mathrm{mGy}$, effective dose= $2.4 \mathrm{mSv}$

and attenuation. When combined with ECG-controlled tube current modulation a dose reduction between $9 \%$ and $26 \%$ can be achieved [14]. However, Tsai et al. [2] believe dose modulation is ineffective in neonates due to the short $\mathrm{CT}$ data acquisition and heterogeneous composition of the bones and soft tissues of the neonatal chest. They also believe that the dose reduction generated by ECG-pulsing is inversely proportional to heart rate, and at $120 \mathrm{bpm}$ the dose saving effect is almost zero.

With each new CT technological development attempts have been made to incorporate new dose saving features, e.g. the deployment of bow-tie filters in cardiac imaging that aggressively restrict radiation beyond the targeted cardiac field-of-view [23], and recently the introduction of iterative reconstruction technique. Assessment of this technique by
Potana et al. $[25,26]$ found significant image noise reduction without loss of diagnostic information on routine chest $\mathrm{CT}$ imaging in adults. On adopting a low-dose imaging technique (with $35 \%$ dose reduction), the iterative reconstructed image quality was found to be similar to a standard dose $\mathrm{CT}$ examination using filtered back projection reconstruction technique.

\section{Radiation dose estimation}

There are no clear standardised guidelines for the estimation of effective dose. This causes significant problems between comparative studies. Methods used include i) Commercially available software (CT EXPO, Stamm \& Nagel, Hannover/Buchholz, Germany) where dose is estimated from actual CT parameters; ii) Using the recorded DLP multiplied by a region specific and age-based coefficient conversion factor, as described by Shrimpton [24].

Of the articles reviewed, centres carrying out paediatric imaging have adopted the latter methodology $[18,27$, 28], whilst Goo \& Yang [19] uses their own formulae, with Jin et al. [20] following Hollingswoth's recommendation [29] by applying coefficient conversion factor of 0.21 .

Ben Saad et al. [5] and Paul et al. [27] have highlighted that the DLP recorded for all body CT examinations on the Siemens (Siemens Healthcare, Forchheim, Germany) system is calculated using a $32 \mathrm{~cm}$ phantom instead of a $16 \mathrm{~cm}$ phantom, which is more suited for calculation in children [30]. Consequently, this causes an under estimation of radiation dose by a factor of 2 in the neonates. Thomas et al. [28] also came to this conclusion in a study carried out on GE Lightspeed equipment (GE, Milwaukee, Wisconsin USA).

\section{Comparison of radiation dose}

An attempt was made to compare the effective dose between the different authors. It was made difficult due to differences in age groups and by incomplete data, with many different methodologies used to estimate the effective dose. A summary of this is set out in Table 1 . This includes our own data from Great Ormond Street Hospital NHS Trust (GOSH).

Radiation burden incurred in MDCT ECG-gated cardiac imaging ranges from 2.17 to $3.41 \mathrm{mSv}$, limiting its use to complex CHD and as a problem solving technique [2]. A retrospective ECG-gated study carried out on a 40-slice MDCT recorded a DLP of 91 mGy.cm (Tsai et al. [6]), this is much higher than centres using a DSCT with reductions of 49/69/76\% recorded by Jin et al. [20], ourselves and Ben Saad et al. [5] respectively. 


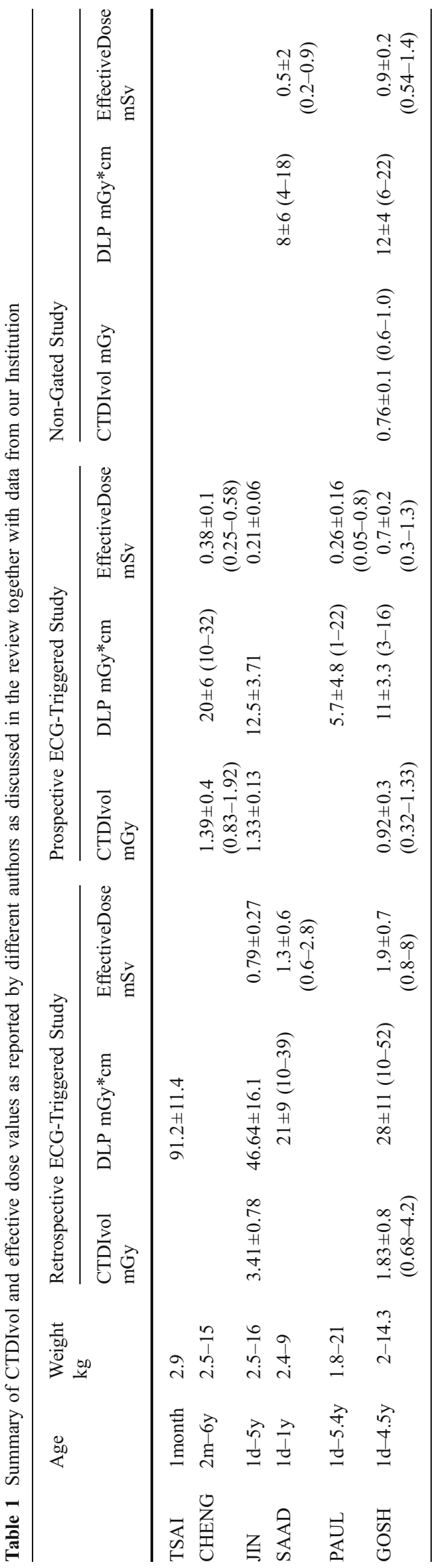

There is a marked dose reduction of $73 \%$ (Jin et al. [20]) and $64 \%$ (our data) when prospective gating is used compared with retrospective gating. The application of prospective gating on a second generation DSCT can achieve further dose reduction as documented by Paul et al. [27] with DLP of 5.7 $\pm 7 \mathrm{mGy} . \mathrm{cm}$ and effective dose of only $0.26 \pm 0.6 \mathrm{mSv}$ (i.e. equivalent annual background dose). This is $71 / 54 / 20 \%$ lower than those recorded by Cheng at al. [18], Jin et al. [20] and at our Institution, respectively.

\section{Future trends}

CT technology is continuously evolving with increasing detector width enabling larger volume coverage, with faster CT data acquisition times, taking cardiac imaging to a new level with the introduction of helical prospective ECGtriggered acquisition at a high pitch.

Newer DSCT equipment allowing a pitch of 3.4 enables gapless volume data acquisition in one cardiac cycle [31]. A $75 \mathrm{~ms}$ temporal resolution is achieved by the use of single-cycle reconstruction. As this is a spiral acquisition, there is smooth transition between each image with no step artefacts demonstrated. The use of the high pitch avoids overlapping slices, thus reducing radiation dose, when compared with the standard sequential step and shoot mode. Radiation dose was found to be significantly lower in helical studies at $0.9 \pm 0.1 \mathrm{mSv}$, as opposed to $1.4 \pm 0.4 \mathrm{mSv}$ in the sequential mode [32].

High pitch CT has also been trialled on early DCST equipment using dedicated software (commercially unavailable as yet) and demonstrated unimpaired image quality with respect to spatial and temporal resolution [33].

A helical prospective ECG-triggered study by DeFrance et al. [34], on a single-source 64-slice MDCT, with automatic adaptation of pitch according to the patient's resting heart rate also demonstrated good quality image when applied to patients with low regular HR (below $65 \mathrm{bpm}$ ) with a reported radiation dose of $6.9 \pm 1.9 \mathrm{mSv}$.

In summary (and from our own experience), prospectively gated cardiac $\mathrm{CT}$ is the preferred technique in young children, where often only morphological and proximal coronary artery detail is required. This negates the need for retrospectively gated imaging with its higher radiation burden. The sharp anatomical delineation between adjacent structures seen in prospective gating is superior to that seen in CTA studies, and with lower radiation dose levels. It is therefore potentially feasible to prospectively ECG-trigger all thoracic imaging to reduce motion artefact and improve overall image quality. 


\section{References}

1. Lee T, Tsai IC, Fu YC (2006) Using multi-detector row CT in neonates with complex congenital heart disease to replace diagnostic cardiac catheterization for anatomical investigation - initial experiences in technical and clinical feasibility. Pediatr Radiol 36:1273-1282

2. Tsai IC, Chen MC, Jan SL, Wang CC, Fu YC, Lin PC, Lee T (2008) Neonatal cardiac multidetector row CT: why and how we do it. Pediatr Radiol 38:438-451

3. Goo HW, Park IS, Ko JK, Kim YH, Seo DM, Yun TJ, Park JJ (2005) Visibility of the origin and proximal course of coronary arteries on on-ECG-gated heart CT in patients with congenital heart disease. Pediatr Radiol 35:792-798

4. Ou P, Celemajer DS, Calcagni G, Brunelle F, Bonnet D, Sidi D (2007) Three-dimensional CT scanning: a new diagnostic modality in congenital heart disease. Heart 93:908-913

5. Ben Saad M, Rohnean A, Sigal-Cinqualbre A et al (2009) Evaluation of image quality and radiation dose of thoracic and coronary dual-source CT in 110 infants with congenital heart disease. Pediatr Radiol 39:668-676

6. Tsai IC, Lee T, Chen MC, Fu YC, Jan SL, Wang CC, Chang Y (2007) Visualisation of neo-natal coronary arteries on multidetector row CT: ECG-gated versus non-ECG-gated technique. Pediatr Radiol 37:818-825

7. Alkadhi H (2009) Radiation dose of cardiac CT-what is the evidence? Eur Radiol 19:1311-1315

8. Arnoldi E, Hohnson TR, Rist C, Wintersperger BJ, Sommer WH, Becker A, Becker CR, Reiser MF, Nikolaou K (2009) Adequate image quality with reduced radiation dose in prospectively triggered coronary CTA compared with retrospective techniques. Eur Radiol 19:2147-2155

9. Stolzmann P, Goetti R, Baumueller S, Plass A, Falk V, Scheffel H, Feuchtner G, Marincek B, Alkadhi H, Leschka S (2010) Prospective and retrospective ECG-gating for CT coronary angiography perform similarly accurate at low heart rates. Eur J Radiol. doi:10.1016/j.ejrad.2009.12.016

10. Wu W, Budovec J, Foley WD (2009) Prospective and retrospective ECG-gating for thoracic CT angiography: a comparative study. AJR 193:955-963

11. Earls J, Berman EL, Urban BA, Curry CA, Lane JL, Jennings RS, McCulloch CC, Hsieh J, Londt JH (2008) Prospectively gated transverse coronary CTA versus retrospectively gated helical technique: improved image quality and reduced radiation dose. Radiology 246(3):742-753

12. Hirai N, Horiguchi J, Fujioka C, Kiguchi M, Yamamoto $H$, Matsuura N, Kitagawa T, Teragawa H, Kohno N, Ito K (2008) Prospective versus retrospective ECG-gated 64-detector coronary CT angiography: assessment of image quality, stenosis and radiation dose. Radiology 248(2):424-430

13. Gopalan D, Raj V, Hoey ETD (2010) Cardiac CT: non-coronary applications. Postgrad Med J 86:165-173

14. Goo HW (2010) State of the art CT imaging techniques for congenital heart disease. Korean J Radiol 11:4-18

15. Blanke P, Bulla S, Baumann T, Siepe M, Winterer JT, Eurynger W, Schaefer O, Kotter E, Langer M, Pache G (2010) Thoracic aorta: prospective ECG-triggered CT angiography with dualsource CT-feasibility and image quality and dose reduction. Radiology 255:207-217

16. Blanke P, Baumann T, Langer M, Pache G (2010) Imaging of pulmonary vein anatomy using low-dose prospective ECG-triggered dual-source computed tomography. Eur Radiol 20:1851-1855

17. Xu L, Yang L, Zhang Z, Li Y, Fan Z, Ma X, Lv B, Yu W (2009) Low-dose adaptive sequential scan for dual-source CT coronary angiography in patients with high heart rate: comparison with retrospective ECG gating. Eur J Radiol 76(2):183-187
18. Cheng Z, Wang X, Duan Y, Wu L, Wu D, Chao B, Liu C, Xu Z, Li H, Liang F, Xu J, Chen J (2010) Low-dose prospective ECG-triggered dual-source CT angiography in infants and children with complex congenital heart disease: first experience. Eur Radiol 20:2503-2511

19. Goo HW, Yang DH (2010) Coronary artery visibility in freebreathing young children with congenital heart disease on cardiac 64-slice CT: dual-source ECG-triggered sequential scan vs. singlesource non-ECG-synchronised spiral scan. Pediatr Radiol 40 (10):1670-1680, Epub 2010 May 13

20. Jin KN, Park EA, Shin CI, Lee W, Chung JW, Park JH (2010) Retrospective versus prospective ECG-gated dual-source CT in pediatric patients with congenital heart disease: comparison of image quality and radiation dose. Int J Cardiovasc Imaging 26:63-73

21. Siegel M (2008) Cardiac CTA: congenital heart disease. Pediatr Radiol 38(suppl 2):S200-S204

22. Goo HW, Park IS, Ko JK et al (2005) Computed tomography for the diagnosis of congenital heart disease in pediatric and adult patients. Int J Cardiovasc Imaging 21:347-365

23. McCollough CH, Primak AN, Saba O, Bruder H, Stierstorfer K, Raupach R, Suess C, Schmidt B, Ohnesorge BM, Flohr TG (2007) Dose performance of a 64-channel dual-source CT scanner. Radiology 243:775-784

24. Shrimpton PC Assessment of patient dose in CT. Chilton, NRPB-PE/ $1 / 2004,2004$. Also published as Appendix C of the 2004 CT Quality Criteria at http://www.msct.eu/PDF_FILES/Appendix\%20paediatric $\% 20 \mathrm{CT} \% 20$ Dosimetry.pdf. Accessed 29th November 2010

25. Potana F, Pagniez J, Flohr T, Faivre J-B, Duhamel A, Remy J, Remy-Jardin M (2010) Chest computered tomography using iterative reconstruction vs filtered back projection (part 1): evaluation of image noise reduction in 32 patients. Eur Radiol. doi:10.1007/s00330-010-1990-5

26. Potana F, Duhamel A, Pagniez J, Flohr T, Faivre J-B, Hachulla A-L, Remy J, Remy-Jardin M (2010) Chest computered tomography using iterative reconstruction vs filtered back projection (part 2): image quality of low-dose CT examination in 80 patients. Eur Radiol. doi:10.1007/s00330-010-1991-4

27. Paul J-F, Rohnean A, Elfassy E, Sigal-Cinqualbre A (2010) Radiation dose for thoracic and coronary step-and-shoot CT using a 128-slice dual-source machine in infants and small children with congenital heart disease. Pediatr Radiol. doi:10.1007/s00247-010-1804-6

28. Thomas K, Wang B (2008) Age-specific effective doses for pediatric MSCT examinations at a large children's hospital using DLP conversion coefficients: a simple estimation method. Pediatr Radiol 38:645-656

29. Hollingsworth CL, Yoshizumi TT, Frush D, Chan FP, Toncheva G, Nguyen G, Lowry CR, Hurwitz (2007) Pediatric cardiac-gated CT angiography: assessment of radiation dose. AJR 189:12-18

30. Huda W, Vance A (2007) Patient radiation doses from adult and pediatric CT. AJR 188:540-546

31. Lell M, Marwan M, Schepis T, Pflederer T, Anders K, Flohr T, Allmendinger T, Kalender W, Ertel D, Thierfelder C, Kuettner A, Ropers D, Daniel WG, Achenbach S (2009) Prospectively ECGtriggered high-pitch spiral acquisition for coronary CT angiography using dual source CT: technique and initial experience. Eur Radiol 19:2576-2583

32. Alkadhi H, Stolzman P, Desbiolles L et al (2010) Low dose, 128slice, dual-source CT coronary angiography: accuracy and radiation dose of the high-pitch and the step-and -shoot mode. Heart 96:933-938

33. Ertel D, Lell MM, Hariq F, Flohr T, Schmidt B, Kalender WA (2009) Cardiac spiral dual-source CT with high pitch: a feasibility study. Eur Radiol 19:2357-2362

34. DeFrance T, Dubois E, Gebow D, Ramirez A, Wolf F, Feuchtner GM (2010) Helical prospective ECG-gating in cardiac computed tomography: radiation dose and image quality. Int $\mathrm{J}$ Cardiovasc Imaging 26:99-107 